\title{
The Executive Function of Temporal Distribution in Time Personality: An ERP (Event-Related Potential) Study*
}

\author{
Guoguang Cao, Yong Zheng \\ Southwest University, Chongqing, China
}

\begin{abstract}
This study investigated the executive function of temporal distribution, which is one of the dimensions of time personality. ERPs (Event-related potentials) were recorded to investigate the temporal patterns of activity while polychronics and monochronics performed a gender word-face Stroop task. Thirty-six participants were selected from 551 valid participants according to scores for the Temporal Distribution subscale of the Time Personality Questionnaire. Polychronics responded faster relative to monochronics in the congruent condition. Moreover, the monochronics elicited relatively larger N170 and sustained positivity amplitude than polychronics, irrespective of task type, but polychronics showed longer latency of N170 relative to monochronics. The findings provide evidence from ERPs for different neural mechanisms in polychronic and monochronic individuals.
\end{abstract}

Keywords: time personality, polychronicity, monochronicity, executive function, word-face task, ERPs (event-related potentials)

\section{Introduction}

In general life and social environments, we find that some people are inclined to do two or more things simultaneously, referred to as polychronics, while others prefer to do one thing at a time, referred to as monochronics. Individuals with such differences in behavioral disposition are likely to perform differently on specific tasks. Temporal distribution is the concept that describes these personality traits. Temporal distribution refers to the behavior and preferences of an individual engaged in one or more tasks within a unit of time. These aspects of personality can be measured by assessments of polychronic switching and polychronic preference. Polychronic switching, which refers to the individual's tendency to switch between multiple tasks, mainly relates to activities at the behavioral level, whereas polychronic preference concerns the experiential level (Lindquist \& Kaufman-Scarborough, 2007; Zhang, Goonetilleke, Plocher, \& Liang, 2005). These two factors together describe the individual differences in temporal distribution.

Temporal distribution is an important dimension and component of time personality. The research into time personality started in the 20th century, and by the end of the 1980s, a large scale study had been conducted. The research addressed many aspects, including an individual's attitude towards time, their experience of it and the related behavior. Kaufman, Lane, and Lindquist (1991) were the first to put forward the concept of time personality to describe an individual's time-using style. A series of subsequent research into time personality

\footnotetext{
* This research was supported by the Doctoral Fund of MOE (20120182110001), China. Guoguang Cao, research assistant, School of Psychology, Southwest University. Yong Zheng, Ph.D., professor, School of Psychology, Southwest University.
} 
focused on behavior (Bluedorn, Kaufman, \& Lane, 1992), and later addressed cognitive and affective factors (Francis-Smythe \& Robertson, 1999). However, recent research has put forward the concept of time motivation in an attempt to reveal the dynamic mechanisms of time personality (Usunier \& Valette-Florence, 2007).

Our studies have found a psychological structure based on time experience and self-concept (Huang \& Zheng, 2000; Zheng \& Huang, 2000), and a four-factor structural model about time personality, including temporal obedience (punctuality and procrastination), temporal programming (management and future-orientation), temporal distribution (polychronic preference and polychronic switching), and temporal tension (impatience and past-orientation) (Chen, 2011). Temporal distribution has always been a research hotspot of time personality. It is an important and relatively stable dimension. Previous research has indicated that polychronics are better able to cope with uncertainty and occupational stress relative to monochronics, and polychronics perform better in interference and switching tasks (Kaufman-Scarborough \& Lindquist, 1999).

Inhibitory control, which is an important part of executive function, refers to the ability to ignore irrelevant stimuli in pursuit of the characterization of cognitive targets. Specifically, inhibitory control refers to a higher cognitive function that involves the exclusion or restraint of interference associated with irrelevant information: The selection of necessary information to be inputted is determined, the relevant information is extracted from long-term memory, and this information is then compared and integrated to enable the inhibition of the dominant response that is unnecessary but has nevertheless been formed. This processing thus leads to coordinated and orderly action and behavior. The ability to control behavior has important significance in terms of achieving success in modern society and in the course of intelligence evolution. The loss or abnormality of the ability to control behavior often leads to conflict and crime (Bjorklund \& Shackelford, 1999; Li, Huang, Constable, \& Sinha, 2006). Research found that the superior medial and precentral frontal cortices were the main neural bases of behavioral control processing ( $\mathrm{Li}$, Huang, Constable, \& Sinha, 2006). Experimental conflict paradigms are considered excellent paradigms to investigate the ability of dynamic executive regulation (Barch, Braver, Carter, Poldrack, \& Robbins, 2009). Cognitive conflict is the one that is related to tasks (e.g., the Stroop task). Cognitive conflict occurs when different representations emerge at the same time and cause interference (Botvinick, Braver, Barch, Carter, \& Cohen, 2001). It is necessary for the brain to monitor the conflict that arises from information processing: the conflict monitoring function. The conflict monitoring system first evaluates the degree of conflict, and then transfers the information to the central nervous system that is responsible for control. The central nervous system then adjusts the current processing leading to conflict resolution. Conflict monitoring and conflict resolution are the key components of cognitive control (Matsumoto \& Tanaka, 2004).

In the field of consumer behavior, one study, which employed investigation and field test methods, concluded that polychronics were inclined to do two or more things simultaneously, whereas monochronics preferred to do one thing at a time (Lindquist \& Kaufman-Scarborough, 2007). In addition, behavioral experiments in the field of computer science found that polychronics and monochronics adopted different control strategies when required to carry out multiple tasks (Zhang, Goonetilleke, Plocher, \& Liang, 2005). Chen (2011) found that polychronics had faster RTs (reaction times) and showed a larger P3 wave than monochronics. These findings all confirmed the theory that polychronics are inclined to do two or more things simultaneously, while monochronics would like to carry out one thing at a time (Zhang et al., 2005; Lindquist \& Kaufman-Scarborough, 2007). Previous studies in the fields of consumer behavior, computer science, and psychology across different cultures have revealed the stable existence of temporal distribution. However, it is still not fully clear what cognitive neural mechanisms underlie the personality traits of temporal distribution. 
Therefore, our study attempted to elucidate the neural mechanisms by employing high temporal resolution ERP (event-related potential) technology.

We used a gender word-face Stroop task to investigate the features of different temporal distribution dispositions during the performance of an inhibitory control task. Using a gender word-face task, previous studies have demonstrated that, following a high level of conflict between task-relevant and task-irrelevant stimuli, attention is more strongly oriented toward target stimuli and away from distractor stimuli (Scerif, Worden, Davidson, Seiger, \& Casey, 2006). We hypothesized that monochronics would require more attention resources, thus enhancing the processing of faces related to the task and inhibiting the processing of words unrelated to the task. The N170 is a negative-going deflection which peaks about $170 \mathrm{~ms}$ after stimulus onset; it is especially pronounced for face stimuli. The N170 probably reflects the processing of structural face encoding in temporo-occipital brain areas and is utilized by subsequent face recognition processes (Eimer, 2000; Kolassa \& Miltner, 2006; Hole \& Bourne, 2010). A top-down attentional amplification in the processing of a predominantly task-relevant face dimension should therefore lead to an elevated N170. The second ERP component that was examined was conflict-N450. The conflict-N450 is a negative-going ERP deflection that is typically observed at central scalp sites. It occurs about $450 \mathrm{~ms}$ after the onset of an incongruent stimulus (Markela-Lerenc, Ille, Kaiser, Fiedler, Mundt, \& Weisbrod, 2004) and probably reflects the processing of conflict monitoring in the anterior cingulate cortex (Badzakova-Trajkov, Barnett, Waldie, \& Kirk, 2009; Bruchmann, Herper, Konrad, Pantev, \& Huster, 2010; Osinsky, Gebhardt, Alexander, \& Hennig, 2012). The last component to be examined was SP (sustained positivity). In the ERP literature this potential has been associated with conflict resolution and has been proposed to play an important role in response selection or in goal maintenance (West, 2003; West, Jakubek, Wymbs, Perry, \& Moore, 2005).

\section{Methods}

\section{Subjects}

Subjects were selected from a pool of 551 Chinese undergraduates or graduates. They were divided into two extreme groups according to the highest and lowest scores for the Temporal Distribution subscale of the Time Personality Questionnaire (Chen, 2011). At the end, 36 subjects aged 17-26 years participated in the experiments for monetary remuneration (18 polychronics ( 8 males) and 18 monochronics (6 males)). The groups differed significantly in terms of the scores of the subscale, $t_{(34)}=9.63, p<0.0001$. They were all right-handed, native Chinese speakers, who were free of any reported affective disorders, and had normal or corrected-to-normal vision. Each participant signed an informed consent form prior to the experiment. The experimental procedure was in accordance with the ethical principle of the 1964 Declaration of Helsinki.

\section{Stimuli}

The stimuli comprised 70 pictures that consisted of 35 male and 35 female neutral facial expressions selected from the Chinese Affective Picture System (Wang \& Luo, 2005). We chose to use neutral facial pictures in order to exclude the interference of emotion. Each facial stimulus was modified in Adobe Photoshop to produce the final experimental material: The Chinese equivalents of the words male/female (“男” or “女”, printed in red color, front size 48) were superimposed onto the center of the noses of male and female faces (width 260 pixels, height 300 pixels). There were 140 picture stimuli in total; both the congruent and incongruent condition had 70 stimuli, with the same amount of male and female faces in each condition. The two conditions were statistically similar in terms of arousal $\left(t_{(15)}=0.06, p>0.05\right)$ and valence $\left(t_{(15)}=-0.02, p>0.05\right)$. 


\section{Task and Procedure}

The experimental paradigm consisted of a computerized version of the gender word-face Stroop task, and included two test conditions (congruent and incongruent) and two blocks (there was a short break between the blocks). A black background was displayed throughout the task. In the behavioral task, which took place in a quiet room, subjects were seated approximately $150 \mathrm{~cm}$ from a computer screen, with the horizontal and vertical visual angles below $6^{\circ}$. Prior to the experiment, all subjects were told that the purpose of the experiment was to investigate their ability to select the facial picture according to the gender. Each trial was initiated by the $300 \mathrm{~ms}$ presentation of a small white cross on a black computer screen. A blank screen was then presented for a duration that varied randomly between $500 \mathrm{~ms}$ and $1,500 \mathrm{~ms}$, and this was followed by the onset of the picture stimulus. In each block, participants were instructed to identify the gender of the face as quickly as possible by pressing the appropriate button ( $\mathrm{F}$ or $\mathrm{J}$ on the keyboard), while ignoring the meaning of the word that was superimposed on the face. The presentation of the stimulus picture was terminated by the pressing of a key, or when the duration of picture stimulus presentation exceeded 1,000 ms. Each response was followed by $1,000 \mathrm{~ms}$ of a blank screen. The response key was counterbalanced across subjects. There was a pre-training session comprising 10 practice trials, which was performed prior to the formal experiment in order to familiarize subjects with the procedure.

\section{Electrophysiological Recording and Analysis}

EEG (electroencephalogram) was recorded from 64 scalp sites using tin electrodes mounted in an elastic cap (Brain Products). The references were placed on the left and right mastoids (average mastoid reference; Luck, 2005) and a ground electrode was positioned on the medial frontal aspect. Vertical EOGs (electrooculograms) were recorded supra- and infra-orbitally at the right eye. Horizontal EOG was recorded from the right orbital rim. EEG and EOG activity was amplified using a DC $\sim 100 \mathrm{~Hz}$ band-pass filter and continuously sampled at $500 \mathrm{~Hz} /$ channel. The EEG was band-pass filtered from 0.01 to $16 \mathrm{~Hz}$. All electrode impedances were maintained below $5 \mathrm{k} \Omega$. ERP averages were computed off-line. Trials with EOG artifacts (mean EOG voltage exceeding $\pm 80 \mu \mathrm{V}$ ) and those contaminated with artifacts due to amplifier clipping, or peak-to-peak deflection exceeding $\pm 80 \mu \mathrm{V}$ were excluded from averaging. EEG activity for correct responses in each condition was overlapped and averaged separately. ERP waveforms were time-locked to the onset of the stimuli and the average epoch was $800 \mathrm{~ms}$, including a $100 \mathrm{~ms}$ pre-stimulus baseline.

We selected the time courses and electrode positions for different ERP components according to the total average ERP waveform and previous electrophysiology research regarding Stroop conflicts. The face specific N170 component, which peaked approximately $170 \mathrm{~ms}$ after stimulus onset, was measured and analyzed at 150-190 ms at sites PO7 and PO8. The SP occurred around 520-620 ms at sites Fz, F1, F2, and FCz. Repeated-measures ANOVAs (analyses of variances) were conducted on the latencies and averaged amplitudes, with Electrode site, Task type and Group as factors. The degrees of freedom for the F-ratio were corrected according to the Greenhouse-Geisser method in all analyses.

\section{Results}

\section{Behavioral Results}

The average accuracy was $92 \%$ for all subjects. The mean RT and accuracy for correct responses, for both groups, are shown in Table 1. In terms of accuracy, the ANOVA indicated that participants were slower on incongruent than congruent trials $(F=46.48, p<0.001)$. There were no other significant main effects or 
interactions. The ANOVA on the RTs revealed significant main effects for Task Type $(F=215.34, p<0.001)$ and Group $(F=46.48, p<0.05)$. There was also a significant interaction between Task type and Group $(F=$ $9.54, p<0.01)$. Investigation of the group effect for each task showed that polychromic individuals responded faster to the congruent task $(F=8.76, p<0.01)$.

Table 1

Reaction Times and Accuracy Rate for Polychronics and Monochronics For Each Task Type $(M \pm S D)$

\begin{tabular}{|c|c|c|c|c|}
\hline & \multicolumn{2}{|c|}{ Polychronicity } & \multicolumn{2}{|c|}{ Monochronicity } \\
\hline & RT (ms) & $\mathrm{ACC}$ & RT (ms) & $\mathrm{ACC}$ \\
\hline Congruent & $525.67 \pm 44.12$ & $0.96 \pm 0.03$ & $576.98 \pm 58.86$ & $0.96 \pm 0.03$ \\
\hline Incongruent & $587.64 \pm 47.30$ & $0.89 \pm 0.05$ & $617.40 \pm 54.81$ & $0.89 \pm 0.08$ \\
\hline
\end{tabular}

Notes. ACC, accuracy rate; RT, reaction time.

\section{Electrophysiological Data}

The repeated-measures ANOVA on average amplitudes of N170 showed a marginal effect of Group $\left(F_{(1,34)}=\right.$ $5.77, p<0.05)$ and a significant main effect of Electrode site $\left(F_{(1,34)}=5.44, p<0.05\right)$. The monochronics elicited a relatively larger N170 than the polychronics irrespective of Task Type, eliciting greater amplitude at PO8 compared with PO7. No other main effects or interactions were found for this component (see Figure 1). In terms of latency, the repeated-measures ANOVA for N170 showed a significant interaction between Group and Electrode site $\left(F_{(1,34)}=9.01, p<0.01\right)$. Investigation of the group effect at each electrode revealed that polychronics showed longer latency of $\mathrm{N} 170$ relative to monochronics at $\mathrm{PO}\left(F_{(1,17)}=5.52, p<0.05\right)$.

Congruent
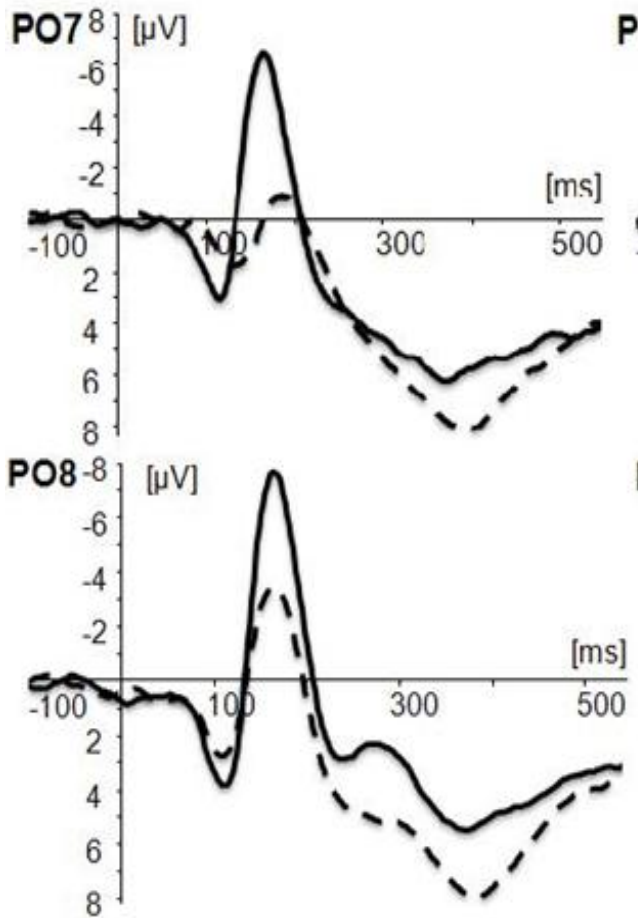

Monoch ronicity
Incongruent
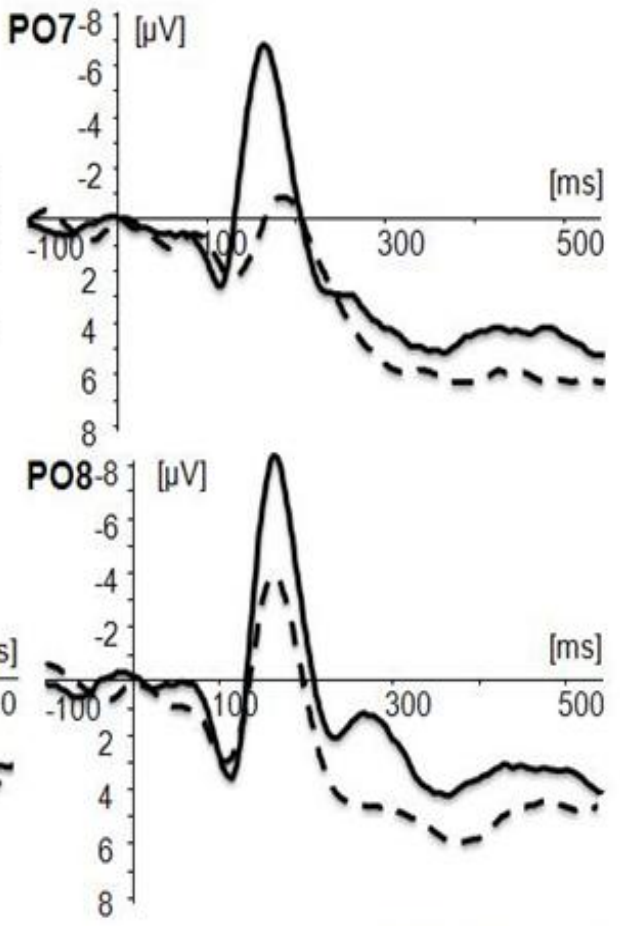

- - - - - Polychronicity

Figure 1. Grand average ERP waveforms of $\mathrm{N} 170$ at PO7 and PO8 for polychronics (dashed lines) and monochronics (solid lines) for different task types. 
For the N450 component, the ANOVA performed on the averaged amplitudes in the 430-480 ms time window showed significant main effects for Task type $\left(F_{(1,34)}=11.50, p<0.001\right)$ and Electrode site $\left(F_{(1,34)}=\right.$ 10.55, $p<0.001)$. There was more negative amplitude elicited for $\mathrm{N} 450$ during incongruent trials than congruent trials. However, there was no significant main effect for Group, and no interaction effects.

Finally, the ANOVA performed on the averaged amplitudes of SP revealed a significant main effects for Group $\left(F_{(1,34)}=4.62, p<0.05\right)$ and Electrode site $\left(F_{(1,34)}=9.93, p<0.001\right)$, which indicated that monochronics elicited larger SPs than polychronics. There were no other main effects or interactions for this component (see Figure 2).

\section{Conguent}

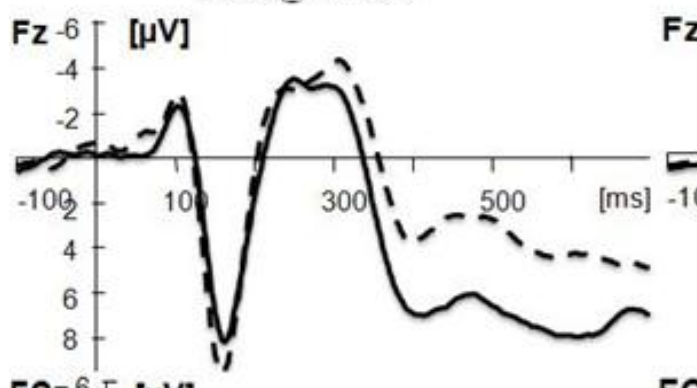

$\mathrm{FCz}^{6}[[\mu \mathrm{V}]$

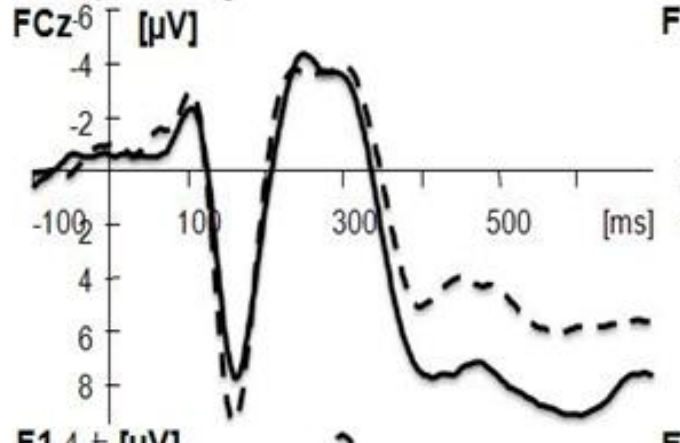

Incongruent

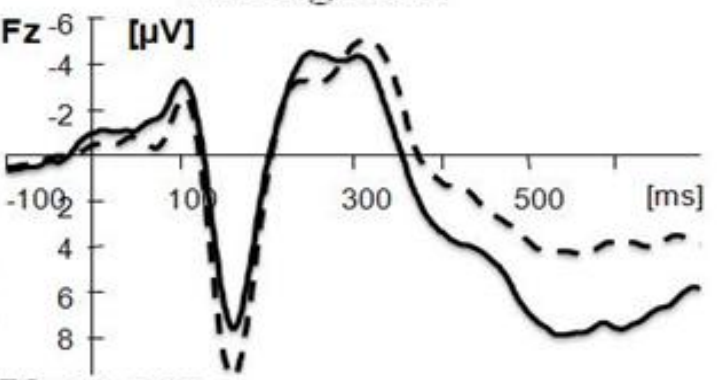

FCz-6 $[[\mu \mathrm{V}]$
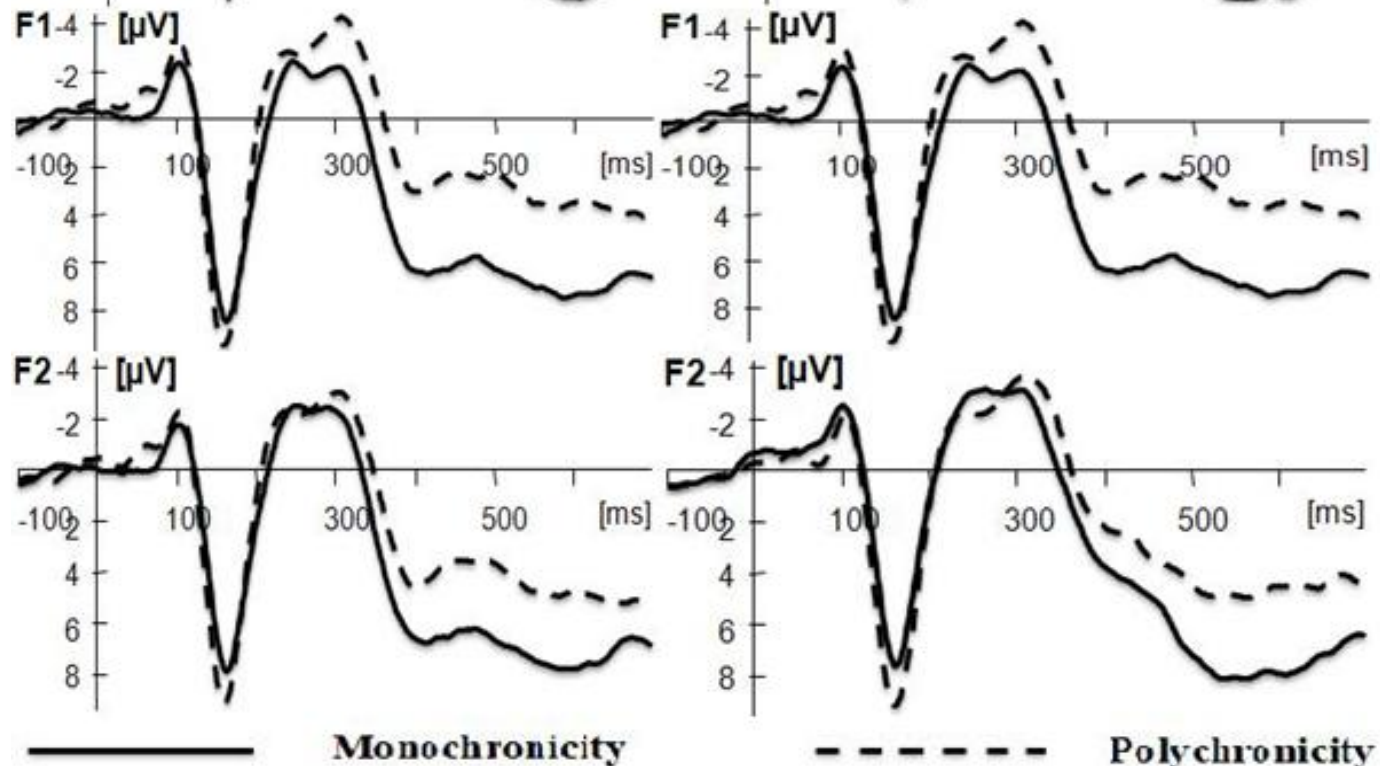

$\mathrm{F} 2-4+[\mu \mathrm{V}]$

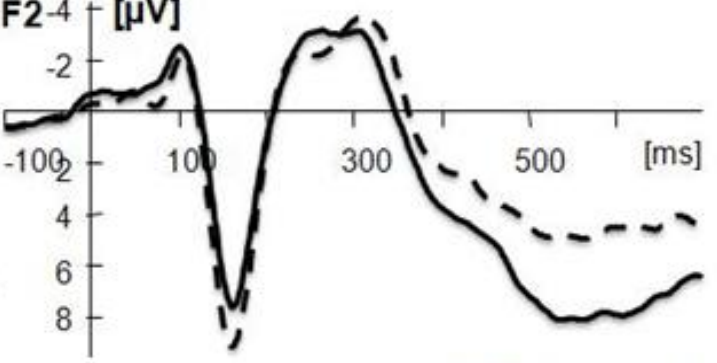

- - - - Polychronicity

Figure 2. Grand average ERP waveforms of sustained positivity at Fz, F1, F2, and FCz for polychronics (dashed lines) and monochronics (solid lines) for different task types. 


\section{Discussion and Conclusions}

This study investigated the time course and activation state of the brain for individuals with different temporal distribution dispositions during performance of a gender word-face task. At the behavioral level, polychronics had significantly faster responses during the task and might inclined to do two or more things simultaneously. The findings suggest that they were able to attend to and judge the gender of the face, while inhibiting the interference associated with the superimposed words. These behavioral results were in line with previous research (Chen, 2011; Lindquist \& Kaufman-Scarborough, 2007; Zhang et al., 2005). However, it was more interesting to find a significant interaction between Task type and Group. Polychronics responded faster to the congruent task than the monochronics. The differences in RT suggest a profound distinction between the groups during the congruent condition. In fact, the RT index contained complex cognitive processes from the onset of the stimuli to the response, including a series of continuous cognitive processes such as perceptual processing, stimulus discrimination, behavior inhibition, and decision-making. We used the high temporal resolution of ERP technology to reveal the time course of the cognitive processes in order to investigate whether group differences existed in each stage of cognitive processing. Specifically, we compared the latency of N170 to examine group differences.

During the 150-190 ms time window, a prominent temporo-occipital N170, reflecting early facial processing, was observed in monochronics. This finding is consistent with that of a previous study (Kolassa \& Miltner, 2006). Greater amplitudes were elicited for the N170 in the right hemisphere (PO8 electrode) compared to the left hemisphere (PO8 electrode). The N170 component elicited during the gender word-face task is accepted as an index of the processing of structural face encoding in temporo-occipital brain areas, and is utilized by subsequent face recognition processes (Eimer, 2000; Hole \& Bourne, 2010; Kolassa \& Miltner, 2006). One might infer that monochronics involved more attention resources in order to complete the task compared to polychronics at the early stages of processing. It was also interesting that, in the current study, polychronics showed longer latency of N170 relative to monochronics at the PO7 electrode site. Polychronics responded faster overall, but this was not the tendency in the early stages of processing. This suggests that polychronics may have an advantage over monochronics during the later stages of cognitive processing. For the monochronics, the earlier attention on the stimulus may have delayed the late cognitive processing, thus prolonging the decision making and executive processing.

This study also observed a significant main effect of task type for the averaged amplitudes of N450. Irrespective of group status, the N450 amplitude was larger during the incongruent condition than the congruent condition. The N450 is a classic ERP component observed during performance of the Stroop task, and reflects the processes of the anterior cingulate cortex (Bruchmann, Herper, Konrad, Pantev, \& Huster, 2010; Osinsky, Gebhardt, Alexander, \& Hennig, 2012; Qiu, Luo, Wang, F. Zhang, \& Q. Zhang, 2006). This finding is consistent with that of a previous study (Qiu et al., 2006). However, there were no other differences between the groups; the conflict monitoring process may be similar for both groups.

About the SP amplitude at the late stage of cognitive processing, which is related to conflict resolution. This sustained positive amplitude plays an important role in response selection or in goal maintenance (West, 2003; West et al., 2005). This study found evidence for enhanced SP amplitude in the monochronics. We can regard the SP as an index of effort making; monochronics may require great effort in order to complete the task effectively, whereas polychronics does not because they do well in the task themselves. 
The findings of the current study provide a significant contribution to the theory of time personality. This study revealed that monochronics elicited prominent N170 and SP amplitudes, but polychronics showed longer latency of N170 relative to monochronics. We found strong neuropsychological evidence that individuals with different temporal distribution dispositions have distinct cognitive processes, and confirmed the stability of temporal distribution.

\section{References}

Badzakova-Trajkov, G., Barnett, K. J., Waldie, K. E., \& Kirk, I. J. (2009). An ERP investigation of the Stroop task: The role of the cingulate in attentional allocation and conflict resolution. Brain Research, 1253, 139-148.

Barch, D. M., Braver, T. S., Carter, C. S., Poldrack, R. A., \& Robbins, T. W. (2009). CNTRICS final task selection: Executive control. Schizophrenia Bulletin, 35, 115-135.

Bjorklund, D. F., \& Shackelford, T. K. (1999). Differences in parental investment contribute to important differences between men and women. Current Directions in Psychological Science, 8, 86-89.

Bluedorn, A. C., Kaufman, C. F., \& Lane, P. M. (1992). How many things do you like to do at once? An introduction to monochronic and polychronic time. The Executive, 6, 17-26.

Botvinick, M. M., Braver, T. S., Barch, D. M., Carter, C. S., \& Cohen, J. D. (2001). Conflict monitoring and cognitive control. Psychological Review, 108, 624-652.

Bruchmann, M., Herper, K., Konrad, C., Pantev, C., \& Huster, R. J. (2010). Individualized EEG source reconstruction of Stroop interference with masked color words. Neuroimage, 49, 1800-1809.

Chen, J. (2011). Researches on time personality with an adaptive orientation (Doctoral dissertation, Southwest University).

Eimer, M. (2000). The face-specific N170 component reflects late stages in the structural encoding of faces. Neuroreport, 11, 2319-2324.

Francis-Smythe, J., \& Robertson, I. (1999). Time-related individual differences. Time \& Society, 8, 273-292.

Hole, G., \& Bourne, V. (2010). Face processing: Psychological, neuropsychological, and applied perspectives. Oxford, UK: Oxford University Press.

Huang, X., \& Zheng, Y. (2000). Self-integration on time perspective: I. projective test for the psychological structure. Acta Psychologica Sinica, 32, 30-35.

Kaufman-Scarborough, C., \& Lindquist, J. D. (1999). Time management and polychronicity: Comparisons, contrasts, and insights for the workplace. Journal of Managerial Psychology, 14, 288-312.

Kaufman, C. F., Lane, P. M., \& Lindquist, J. D. (1991). Exploring more than 24 hours a day: A preliminary investigation of polychronic time use. Journal of Consumer Research, 18, 392-401.

Kolassa, I. T., \& Miltner, W. H. (2006). Psychophysiological correlates of face processing in social phobia. Brain Research, 1118, $130-141$.

Li, C. R., Huang, C., Constable, R. T., \& Sinha, R. (2006). Imaging response inhibition in a stop-signal task: Neural correlates independent of signal monitoring and post-response processing. The Journal of Neuroscience, 26, 186-192.

Lindquist, J. D., \& Kaufman-Scarborough, C. (2007). The polychronic-monochronic tendency model: PMTS scale development and validation. Time \& Society, 16, 253-285.

Markela-Lerenc, J., Ille, N., Kaiser, S., Fiedler, P., Mundt, C., \& Weisbrod, M. (2004). Prefrontal-cingulate activation during executive control: Which comes first? Cognitive Brain Research, 18, 278-287.

Matsumoto, K., \& Tanaka, K. (2004). The role of the medial prefrontal cortex in achieving goals. Current Opinion in Neurobiology, 14, 178-185.

Osinsky, R., Gebhardt, H., Alexander, N., \& Hennig, J. (2012). Trait anxiety and the dynamics of attentional control. Biological Psychology, 89, 252-259.

Qiu, J., Luo, Y., Wang, Q., Zhang, F., \& Zhang, Q. (2006). Brain mechanism of Stroop interference effect in Chinese characters. Brain Research, 1072, 186-193.

Scerif, G., Worden, M. S., Davidson, M., Seiger, L., \& Casey, B. J. (2006). Context modulates early stimulus processing when resolving stimulus-response conflict. Journal of Cognitive Neuroscience, 18, 781-792.

Usunier, J. C., \& Valette-Florence, P. (2007). The time styles scale: A review of developments and replications over 15 years. Time \& Society, 16, 333-366. 
Wang, Y., \& Luo, Y. (2005). Standardization and assessment of college students' facial expression of emotion. Chinese Journal of Clinical Psychology, 13, 396-398.

West, R. (2003). Neural correlates of cognitive control and conflict detection in the Stroop and digit-location tasks. Neuropsychologia, 41, 1122-1135.

West, R., Jakubek, K., Wymbs, N., Perry, M., \& Moore, K. (2005). Neural correlates of conflict processing. Experimental Brain Research, 167, 38-48.

Zhang, Y., Goonetilleke, R. S., Plocher, T., \& Liang, S. F. M. (2005). Time-related behaviour in multitasking situations. International Journal of Human-computer Studies, 62, 425-455.

Zheng, Y., \& Huang, X. (2000). Self-integration on time perspective: II. experimental research for the psychological mechanism. Acta Psychologica Sinica, 32, 36-39. 